\title{
Monozygotic twins discordant for Wiedemann-Beckwith syndrome and the implications for genetic counselling
}

\author{
A CAROLINE BERRY, E M BELTON, AND C CHANTLER \\ From the Department of Paediatrics and the Paediatric Research Unit, Guy's Hospital Medical School, \\ London Bridge, London SE1 9RT; and St Peter's Hospital, Chertsey, Surrey
}

SUMMARY A pair of monozygous twins discordant for Wiedemann-Beckwith syndrome is described and published reports reviewed to establish the mode of inheritance. Single gene control seems $\vec{\omega}$ unlikely and it is proposed that the condition arises from the interaction of 'at risk' genes in the fetus and metabolic factors in the mother, which suggests multifactorial control.

The Wiedemann-Beckwith syndrome (WBS), also called exomphalos-macroglossia-gigantism syndrome, was first described in 1964 by Wiedemann, ${ }^{1}$ and his reported family included three affected sibs. Irving $^{2}$ also described two affected sibs and after a review of published reports in 1970, Filippi and McKusick $^{3}$ proposed that the condition behaved in an autosomal recessive manner. Reports since then have been more in keeping with autosomal dominant inheritance, ${ }^{4-6}$ while recently Sommer et al $^{7}$ postulated 'delayed mutation' to explain their findings, though in 1974 Lubinsky et $a l^{8}$ had used the term premutation to explain transmission in similar families. Wiedemann ${ }^{9}$ and Gardner ${ }^{10}$ both thought that multifactorial control best explained the familial occurrences.

We wish to report a pair of twins, highly likely to be monozygous, one of whom is entirely normal while the other has the syndrome's classical features, excluding exomphalos, but including the hypoglycaemia and renal medullary dysplasia now recognised as important parts of this syndrome.

\section{Case report}

The proband (PRU 11299) was the first of twin girls delivered normally at 37 weeks' gestation. The parents, who are healthy and not consanguineous, have two healthy older children.

At birth a single placenta was noted. The proband weighed $3760 \mathrm{~g}$ and her twin $2780 \mathrm{~g}$, but in spite of being the heavier the proband required 30 minutes Received for publication 8 May 1979 intubation. She was noted to have a protuberant $\vec{\emptyset}$ abdomen with a mass in the left hypochondrium. IVU showed possible bilateral multicystic kidneys.

At the age of 7 weeks she was admitted to her local hospital semiconscious and with convulsions shown to be secondary to hypoglycaemia. Macroglossia and hemihypertrophy were now present and $\mathbb{D}$ WBS was diagnosed. Repeat IVU showed evidence of medullary dysplasia and possible vesicoureteric 윽 reflux with dilated tortuous ureters and she was admitted to Guy's Hospital for further investigation. The hypoglycaemia was being satisfactorily controlled with diazoxide.

At the age of 12 weeks her length was $61 \mathrm{~cm}, \frac{0}{0}$ weight $5560 \mathrm{~g}$. She had a broad flat-bridged nose with 3 . large protuberant tongue. Her ear lobes did not $\varnothing$ show the indentations frequently noted in patients with this syndrome. Her limbs were of normal shape but those on the left were considerably larger than those on the right. The cardiovascular system was $\frac{D}{0}$ normal, as were the genitalia. She had a large floppy abdomen but no umbilical hernia, and her kidneys $N$ were just palpable. There was no evidence of $N$ developmental delay.

Her twin had no abnormal physical signs, and her ${ }_{0}^{\infty}$ ear lobes showed no indentations.

Routine investigation gave normal results for blood glucose, creatine, and creatinine clearance.

A micturating cystogram showed grade 3 reflux and at the age of 4 months $\mathrm{Mr} \mathrm{C} \mathrm{H}$ Kinder performed bilateral reimplantation of the ureters.

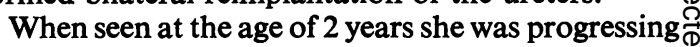
normally and had normal renal function though the $\alpha$ 
TABLE Zygosity determination

\begin{tabular}{llllllllll}
\hline Marker & $P$ & $A D A$ & $E S D$ & $A B O$ & $M N$ & $S s$ & $R h$ & $L e^{\mathrm{a}} L e^{\mathrm{b}} J k^{\mathrm{a}} J k^{\mathrm{b}}$ \\
\hline Mother + & $\mathbf{1}$ & $\mathbf{1}$ & $\mathbf{O}$ & $\mathbf{M N}$ & -+ & $\mathbf{r r}$ & -+ & ++ \\
Father + & $2-1$ & $2-1$ & $\mathbf{A}_{1}$ & $\mathbf{M}$ & ++ & $\mathbf{R}_{2} \mathbf{r}$ & $-/$ & ++ \\
Twin 1 & + & $2-1$ & $2-1$ & $\mathbf{A}_{1}$ & $\mathbf{M}$ & ++ & $\mathbf{r r}$ & $-/$ & -+ \\
Twin 2 & + & $2-1$ & $2-1$ & $\mathbf{A}_{1}$ & $\mathbf{M}$ & ++ & $\mathbf{r r}$ & $-/$ & -+
\end{tabular}

Systems tested but uninformative: PGM, Hp, Tf, PGD, AK, Gc, $\mathbf{K m}, \mathbf{G O T}_{\mathrm{s}}, \mathrm{Pi}$, blood groups: Lutheran, Kell, Duffy, $\mathbf{X g}^{\mathrm{a}}$.

left ureter remained dilated. Vesicoureteric reflux was no longer present and both kidneys were growing with normal renal function.

INVESTIGATIONS TO ESTABLISH ZYGOSITY Blood group and enzyme studies were carried out by staff of the MRC Human Biochemical Genetics Unit and of the MRC Blood Group Unit. The results are shown in the table and the final probability of monozygosity is $\mathbf{0 . 9 9 5}$.

\section{Discussion}

The occurrence of this syndrome in one of a pair of monozygotic twins is, for practical purposes, incompatible with single gene control. Undoubtedly, familial cases do occur and the reported families strongly suggest that genetic factors are operative. The reports of an affected parent and child ${ }^{6}$ point towards a dominant pattern of inheritance, but in several families where such inheritance has been suggested, ${ }^{4511}$ a large number of clinically normal gene carriers have to be postulated, and this suggests that more than one factor must be responsible. Delayed mutation, as suggested by Sommer et $a l,{ }^{7}$ involves two mutational steps. Subjects carrying the premutated gene are normal themselves, but have affected progeny when the second or telomutation occurs. This theory would predict that both of monozygotic twins would be affected unless the telomutation occurred in very early embryonic life.

The occurrence of affected family members in more than one generation, as well as the birth of several affected children to apparently normal parents and the discordance of MZ twins, suggests that multifactorial control is most likely. What factors are likely to be important?

The sex ratio of reported cases is unremarkable ( 37 males, 43 females) so the sex of the child seems uninfluential.

Minor stigmata, such as ear lobe indentation, have occasionally been noted in unaffected family members ${ }^{6}{ }^{10}$ and this suggests that the genetic background is important, so that the presence of certain alleles at one or several susceptibility loci might be necessary for the development of the condition.

In the families described by Irving, ${ }^{2}$ Kosseff $e t a l,{ }^{4}$
Forrester, ${ }^{5}$ and Sommer et al, ${ }^{7}$ the affected children were all born to female family members (a point first noted by Lubinsky et $a l^{8}$ ) and this raises the possibility of a familial maternal environmental effect. This idea, however, is complicated by the family reported by Matsuura et $a^{11}$ in which the three affected members are related through their fathers. Several authors ${ }^{6} 12$ have noted the occurrence of diabetes mellitus in families with members with WBS, so that altered carbohydrate metabolism might be a precipitating maternal factor. Two mothers who had glucose tolerance tests gave normal results, ${ }^{3}$ but this test is a comparatively crude tool. We have noted that three consecutive families referred here have a family history of twinning. One pair is reported here, the next child was one of twins, the other dying in utero, and in the third there were several pairs of twins in both parental families. This association has not been apparent in other reported families, but it is possible that factors predisposing to twinning could also predispose to the development of WBS.

It could be argued that monozygous twins should be equally affected by the maternal environment and hence be concordant, but discordancy of monozygous twins is a recognised phenomenon in multifactorially controlled conditions and probably reflects, for example, differences in blood supply to the individual twins.

We believe that the evidence from this and other reported families supports Gardner's ${ }^{10}$ concept that the interaction of at risk genes in the fetus and metabolic factors in the mother gives rise to this syndrome with its omphalocele hemihypertrophy, gigantism, and susceptibility to hypoglycaemia and malignancy, mediated perhaps by hypothalamic dysfunction.11

At a more practical level accurate genetic counselling for these families is difficult. Many cases appear to be sporadic and the 1 in 4 recessive recurrence risk seems too high. There is a clear need for family studies to establish empirical risk figures, but until these are available counselling appropriate for multifactorial control seems reasonable.

Professor E B Robson and Dr R Sanger gave assistance with the zygosity determination, for which we are very grateful. We thank Professor P E Polani for encouragement to publish this report and acknowledge financial support from the Department of Health and Social Security and the Spastics Society.

\section{References}

1 Wiedemann H-R. Complexe malformatif familial avec 
hernie ombilicale et macroglossie: un 'syndrome nouveau'? J Genet Hum 1964;13:223-32.

2 Irving IM. Exomphalos with macroglossia: a study of eleven cases. J Pediatr Surg 1967;2:499-507.

3 Filippi G, McKusick VA. The Beckwith-Wiedemann syndrome. (The exomphalos-macroglossia-gigantism syndrome): report. Medicine 1970;49:279-98.

4 Kosseff AL, Hermann J, Opitz JM. The WiedemannBeckwith syndrome: genetic considerations and a diagnostic sign. Lancet 1972;1:844.

5 Forrester RM. Wiedemann-Beckwith syndrome. Lancet 1973;2:47.

- Ben-Galim E, Gross-Kieselstein E, Abrahamov A. Beckwith-Wiedemann syndrome in a mother and her son. Am J Dis Child 1977;131:801-3.

7 Sommer A, Cutler EA, Cohen BL, Harper D, Backes C. Familial occurrence of Wiedemann-Beckwith syndrome and persistent fontanel. Am J Med Genet 1977;1:59-63.

8 Lubinsky M, Herrmann J, Kosseff AL, Opitz JM. Auto- $\frac{\mathbb{D}}{\overparen{D}}$ somal-dominant sex-dependent transmission of the $\stackrel{\mathbb{P}}{?}$ Wiedemann-Beckwith syndrome. Lancet 1974;1:932.

9 Wiedemann H-R. E.M.G. syndrome. Lancet $1973 ; 2: 626$.

10 Gardner LI. Pseudo-Beckwith-Wiedemann syndrome: 0 interaction with maternal diabetes. Lancet 1973;2:911-2.

11 Matsuura N, Endo M, Okayasu T, Okuno A. WiedemannBeckwith syndrome. Lancet 1975;2:508.

12 Cohen MM, Gorlin RJ, Feingold M, Ten Bensel RW. ¿ The Beckwith-Wiedemann syndrome. Am $J$ Dis Child के $1971 ; 122: 515-9$.

Requests for reprints to Dr A C Berry, Paediatric $\omega_{\mathscr{\sigma}}$ Research Unit, Guy's Hospital Medical School, London Bridge, London SE1 9RT. 